\title{
Mild Normobaric Hypoxia Exposure for Human-Autonomy System Testing
}

\author{
Chad L. Stephens \\ NASA Langley \\ Research Center
}

\author{
Kellie D. Kennedy \\ NASA Langley \\ Research Center
}

\author{
Brenda L. Crook \\ Analytical Mechanics \\ Associates, Inc.
}

\author{
Ralph A. Williams \\ Analytical Mechanics \\ Associates, Inc.
}

Paul Schutte

U.S. Army

\begin{abstract}
An experiment investigated the impact of normobaric hypoxia induction on aircraft pilot performance to specifically evaluate the use of hypoxia as a method to induce mild cognitive impairment to explore human-autonomous systems integration opportunities. Results of this exploratory study show that the effect of 15,000 feet simulated altitude did not induce cognitive deficits as indicated by performance on written, computer-based, or simulated flight tasks. However, the subjective data demonstrated increased effort by the human test subject pilots to maintain equivalent performance in a flight simulation task. This study represents current research intended to add to the current knowledge of performance decrement and pilot workload assessment to improve automation support and increase aviation safety.
\end{abstract}

\section{INTRODUCTION}

An experiment was conducted to study the effect of normobaric hypoxia induction on aircraft pilot cognition, task performance, and workload. Specifically, the purpose of this study was to investigate the use of hypoxia as a method to induce mild cognitive impairment during simulated flight and other tasks to permit future studies of human-autonomous systems integration. The intent being that future autonomous system may incorporate continuous monitoring and assess of pilot mental and physical state and respond appropriately when cognitive impairment is detected. Data collected can be used to support development of automation response algorithms designed to mitigate consequences of cognitive and physiological deficit.

Hazardous States of Awareness in Aviation. Human error (Rasmussen, 1982) induced by automation in complex contexts has been studied from multiple perspectives, including a focus on Hazardous States of Awareness (HSAs) detectable by psychophysiological measurements (Pope \& Bogart, 1992). In empirical studies of HSAs, whether they be in laboratory or simulated environments, there are conventions relied upon to induce these states (Stephens, Scerbo, \& Pope, 2012). These conventions are intended to mimic reality including: black swan events, individual or team confusion due to malfunctioning equipment/mechanical failure, crew confusion due to lack of understanding/unfamiliarity, over engagement in secondary tasks, and/or leveraging tendency of poor monitoring for extended periods of time coupled with non-normal scenarios (Parasuraman, Molloy, \& Singh, 1993; Scerbo, 1996; Wickens, Hooey, Gore, Sebok, \& Koenicke, 2009). While these methods have been effective in experimental contexts, opportunity remains for novel methods of inducing error to be developed.

Hypoxia as a Proxy for HSAs. A physiological response which has been studied extensively and results in impairment of the central nervous system is hypoxia, defined as deprivation of adequate oxygen supply at the bodily tissue level. Hypoxia has been studied in the aviation context because increasing altitude results in decreased gas pressure and consequently oxygen available to breathe. The general impact of hypoxia on mental experience is referred to as Time of Useful Consciousness (TUC; DeHart \& Davis, 2002) also termed Effective Performance Time (EPT; Pickard, 2002). TUC and EPT reference charts indicate the average amount of time a human can remain conscious at increasing altitudes and remain capable of completing tasks respectively.

Cognitive Impairment in Humans Due to Hypoxia.

Previous research has shown there can be substantial individual variability in tolerances to hypoxia (Self, Mandella, White \& Burian, 2013). In general, hypoxia's effects on performance follow a dose-response trend as does the cardiopulmonary protective response effectiveness (Harding \& Mills, 1983; Woodrow \& Webb, 2011). This trend is captured in the previously mentioned TUC reference charts. Perhaps more cogent to aviation operations, TUC should be regarded as the time during exposure to a hypoxic environment in which the aviator is still able to correct the situation (Gradwell, 2006). Historically, TUC tables were based upon hypobaric research, typically beginning at 18,000 feet with an EPT of 20 to $30 \mathrm{~min}$, before which the compensatory cardiorespiratory mechanisms provide some protection and extend functionality (DeHart \& Davis, 2002). Tables describing TUC and EPT would end at 50,000 feet with an TUC/EPT of 9 to 12 seconds, representing both a point of net oxygen diffusion out of the pulmonary capillaries as well as the atmospheric region where full pressure suits are required not to just maintain adequate pressure-based oxygenation but also to prevent severe decompression sickness. (FAA AC 61107B; Pickard, 2002; Woodrow \& Webb, 2011). However, more recently, sophisticated human respiratory system modeling has been proposed that may provide a more precise decompression scenario-based degree of impairment by hypobaric hypoxia exposure (Wolf, 2014).

Cognitive assessment tools that map well to aviation performance have mixed results in hypoxia studies. During tests using the Multi-Attribute Task Battery (MATB), Swain 
et al (1999) found no significant performance effects of exposure to $10,000,15,000,20,000$ or 25,000 feet on the Fuel management, communications, or systems monitoring subtask responses. Swain et al. did find significant increases in reaction time response to the lights of the systems monitoring task as well as increases in error during the tracking task but only at 25,000 feet. Interestingly, they were able to positively correlate increases in electroencephalogram (EEG) alpha and theta activity with decreased performance on systems monitoring and tracking tasks, respectively. Nesthus, Garner \& Mills (1997) found no significant effects for altitude on MATB Performance for non-smokers at 5,000, 8,000 and 12,500 feet. Hewett, Curry \& Gaydos (2010) used the CogScreen-Hypoxia Edition (CogScreen-HE) to assess cognitive changes at Sea Level, 8,000, 10,000, 12,000 and 14,000 feet and found no significant change in reaction time, accuracy or throughput on any of the battery of tests.

However, Rice et al (2005) found a significant decrement in accuracy during the CogScreen-HE Vigilance subtest but only at 15,000 feet.

During flight simulator studies, hypoxia leads to increased procedural errors during descent and landing phases in general aviation flight from altitudes of 10,000 and 12,500 feet as well as increased subjective ratings of temporal demand on NASA Task Load Index (NASA-TLX) subjective workload ratings (Nesthus, Rush \& Wreggit, 1997). Increased altitude and airspeed variance for maintaining constant 3,000 feet, 180 degree heading, and 70 knot airspeed in flight simulator was found at 18,000 feet normobaric hypoxia (Temme, Still \& Acromite, 2010).

Given the effects of mild hypoxia on cognitive function the current study was undertaken to assess whether controlled hypoxia exposure could serve as a proxy for HSAs. The 10 minute exposures at 15,000 feet were selected for this exploratory study because this simulated altitude is low enough to permit repeated exposure safely. Furthermore, it was expected that this normobaric altitude exposure would induce cognitive deficits due to the typical human subject's cardiorespiratory compensatory limits. Reported herein are the methods, results, and discussion of findings from an exploratory study involving mild normobaric hypoxia exposure in human test subjects performing a variety of laboratory and flight simulation tasks.

\section{Experimental Design}

\section{METHOD}

The design of the experiment included exposure to a Reduced Oxygen Breathing Device mark 2 (ROBD2) equivalent altitude (i.e., normobaric hypoxia induction) at two Levels: Sea Level $\left(21 \% \mathrm{O}_{2}\right)$ and 15,000 feet $\left(11.2 \% \mathrm{O}_{2}\right)$. Both of these normobaric altitudes were experienced by the test subjects while they completed three separate tasks.

\section{Experimental Protocol}

Tasks. Three 40 minute sessions (three 10 minute runs with recovery periods following each run) involving written
Cognitive Function Test (CFT; Westermann, 2004) or CogScreen-HE (COG, Kay, 1995), computer-based multi-task battery (MATB), or flight simulation (FLT) tasks. Three runs of each task: Training (room air), then single-blind exposure to Sea Level, and 15,000 feet. Half of the test subjects completed the CFT and the other half completed the COG. Both of these cognitive skills tests required the same 40 minutes to complete and were the first task that all test subjects experienced when participating in the study. The MATB and FLT task order was counter-balanced.

Task Training. Subjects completed 10 minutes of instruction and practice immediately prior to completing each of the three tasks. Ten minutes of instruction on flight simulator, pre-flight brief, and practice in completing the FLT task occurred prior to completion of the full FLT task. The subjects were instructed to maintain the appropriate airspeed within \pm 10 knots, maintain altitude within \pm 100 feet, and accurately track a heading within $\pm 10^{\circ}$.

Hypoxia Manipulation. Each test subject breathed room air for the first 10 minutes while completing training on each task. The subsequent performance of each task was at Sea Level or 15,000 feet for 10 minutes, subject performed the task while breathing a gas mixture from the ROBD2.

Apparatus. The study was conducted in the NASA Langley Research Center (LaRC) Human and Autonomous Vehicle Systems (HAVS) Laboratory and involved the use of an Environics, Inc. ROBD2 Model 6202. The ROBD2 is a computerized gas-blending instrument used to generate hypoxic air without changes in atmospheric pressure (see Figure 1). Each test subject wore a Gentex MBU-20/P aviator's oxygen mask connected to the ROBD2 during the experimental session. Following each Sea Level and 15,000 feet exposure test subjects recovered on Sea Level or $100 \% \mathrm{O}_{2}$ for 2 minutes respectively, until $\mathrm{SPO}_{2}$ returned to $98 \%$ and all self-reported hypoxia symptoms were absent. Subjects completed the NASA-TLX (using the unweighted procedure) self-reported measure of subjective workload after each task session. Additionally, test subjects completed a Post-Run Questionnaire including self-report of hypoxia symptoms.

At the end of the experiment, test subjects completed a Post-Experiment Questionnaire which capture more detail about their experience during each of the task runs. Subjects were fully debriefed following final session including: revealing which were 15,000 feet runs, discussion of subject's hypoxia response \& task performance, and discussion of aftereffects of hypoxia and safety measures.

\section{Participants}

Subject Recruitment and Eligibility. Professional pilots (commercial and military) served as test subjects $(n=57,49$ males). Their age ranged from 22 to 60 years $(M=41.4, S D=$ 9.2). Five participants were excluded from analyses due to missing data. All test subjects received compensation in the form of a stipend and coverage for travel costs. 
Extensive eligibility requirements were instituted in order for subjects to participate in the study including U.S.

Citizenship, at least 20 years of age and not older than 60 years of age, held valid Aeromedical Certificate Class I, Class II, or military equivalent at the time of the study. Also, subjects were required to have previously completed Hypoxia Recognition Training (HRT) involving exposure to hypoxia in a barochamber or an ROBD, and a copy of the HRT completion certificate or a signed self-verification of HRT provided. Eligible subjects could not be prone to motion sickness per the Simulator Sickness pre-screening Questionnaire, did not self-report any major body system medical conditions. A medical screening was completed on all test subjects at the NASA LaRC Health Clinic. Three medical tests were performed and all results were required to be within normal limits; tests were: a resting electrocardiogram (EKG), pulmonary function test, and a Complete Blood Count (CBC) with Differential (first 28 test subjects) or Point-of-Care Anemia test (last 29 test subjects). This study received approval from the Institutional Review Board at NASA LaRC.

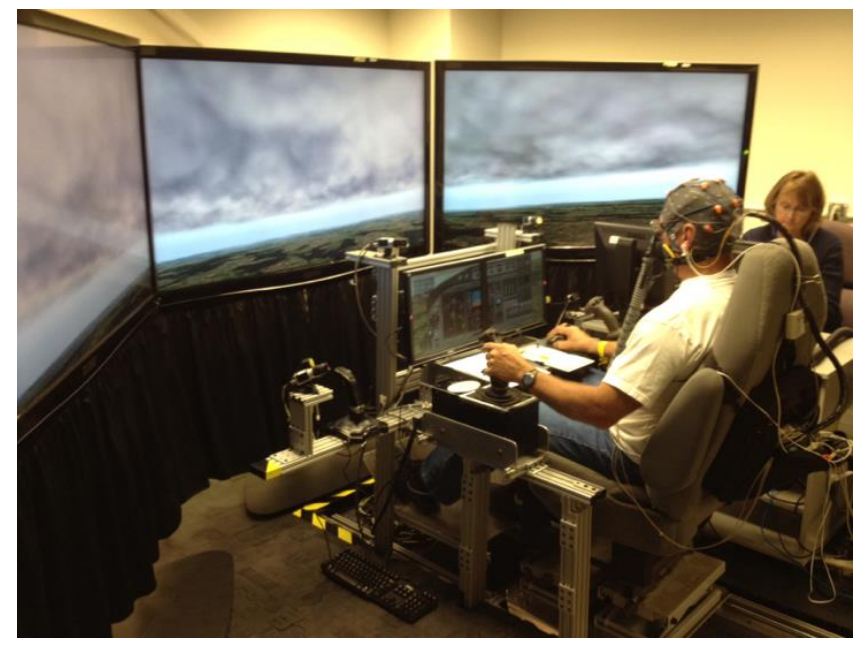

Figure 1. Test Subject in NASA LaRC HAVS Lab.

\section{Dependent Variables}

Self-report measures were employed to capture symptoms experienced during each session via the post-run questionnaire and post-experiment questionnaire, both of which were multiple choice and open response. Workload was assessed via the NASA-TLX. Objective Performance measures included: Cognitive Function Test scores on simple computation, serial subtraction, eye-hand coordination, semantic memory, visual-motor coordination, short-term memory, graphic memory, and coordination; and CogScreenHE sub-tests scores indicating impact of hypoxia on visual attention, working memory, verbal-sequencing processing, visual-perceptual speed, visual-motor speed, divided attention, capacity for multitasking, visual scanning, speed of information processing, number and letter sequencing skills, ability to systematically apply an organizing principle, immediate memory, motor coordination, and ability to shift mental set (Kay, 1995). The MATB-II Figure of Merit (FOM) score was automatically tabulated by the software and provided FOM overall score and FOM individual task score. The Flight Simulation (FLT) task performance was assessed by an algorithm developed to calculate flight technical error.

Cortical and physiological measures included: 1 ) saturation of peripheral oxygen $\left(\mathrm{SPO}_{2}\right)$, pulse rate, respiration rate as hypoxia induction manipulation checks; 2) EEG to measure change in predominance of high (beta) and low (alpha \& theta) frequency activity; 3 ) Electrocardiogram (EKG) to permit Heart Rate Variability (HRV) analysis, specifically frequency domain indicators of stress and underload; 4) Galvanic Skin Response (GSR) to assess sympathetic nervous system/fight-or-flight response.

\section{RESULTS}

Manipulation check. $\mathrm{SPO}_{2}$ changes were significantly different $(p<0.05)$ based on t-test analyses between the Sea Level and 15,000 feet simulated altitude conditions for all task conditions (see Figure 2). Heart rate changes were not significantly different between the two conditions (see Figure 3).

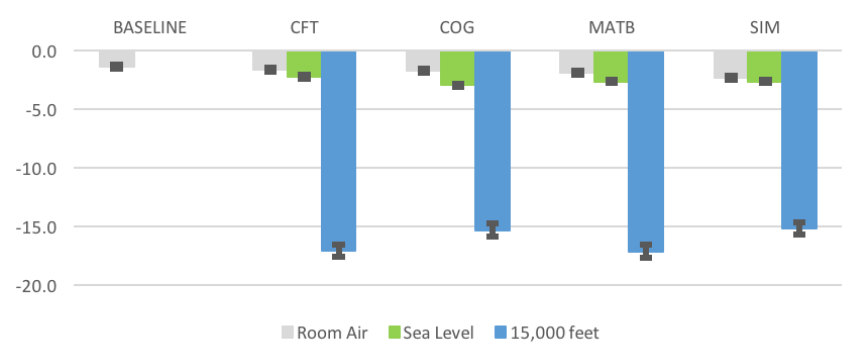

Figure 2. Average $\mathrm{SPO}_{2}$ change due to room air and equivalent altitude exposure - sea level and 15,000 feet.

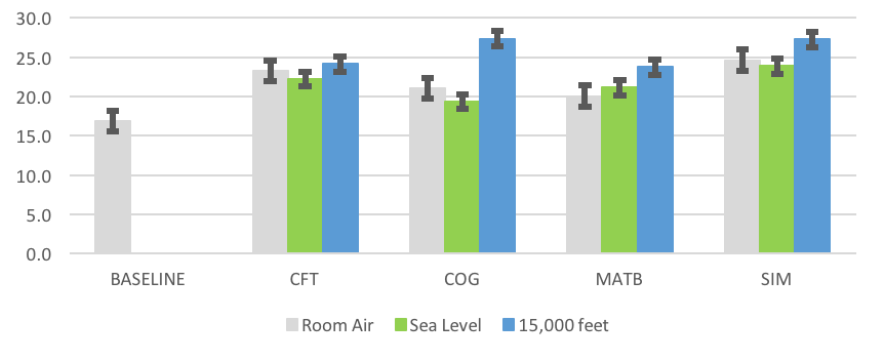

Figure 3. Average Heart Rate change due to room air and equivalent altitude exposure - sea level and 15,000 feet.

Self-Reported Workload. Self-Reported Workload for Sea Level vs 15,000 feet during CFT, CogScreen, and MATB were non-significant $(p>0.05)$. Statistically significant $(p<$ 0.05 ) differences were found in the self-reported NASA-TLX workload between the two altitude conditions for the flight simulation task (See Figure 4). NASA-TLX Overall Score was significantly different $\mathrm{t}(52)=1.8136, \mathrm{p}=0.0036$. NASA-TLX Mental Demand was significantly different $\mathrm{t}(52)=1.1726, \mathrm{p}=$ 0.0488 . NASA-TLX Performance was significantly different $\mathrm{t}(52)=2.668, \mathrm{p}=0.0412$. NASA-TLX Frustration was significantly different $\mathrm{t}(52)=2.189, \mathrm{p}=0.0154$. 
Task Performance. ANOVA statistical testing revealed nonsignificant $(p>0.05)$ differences in performance scores on the three types of tasks (CFT/COG, MATB, FLT) between the two altitude conditions.

Physiological Responses. ANOVA statistical testing of EEG variables derived from frequency band powers in the alpha, theta, or beta bandwidths, revealed no statistically significant differences between Sea Level and 15,000 feet conditions during performance of any of the tasks. Furthermore, ANOVA statistical analysis of the Engagement Index (EI; Pope, Bogart, \& Bartolome, 1995) derived from EEG indicated no significant differences between Sea Level and 15,000 feet conditions during performance of any of the task (See Figure $5)$.

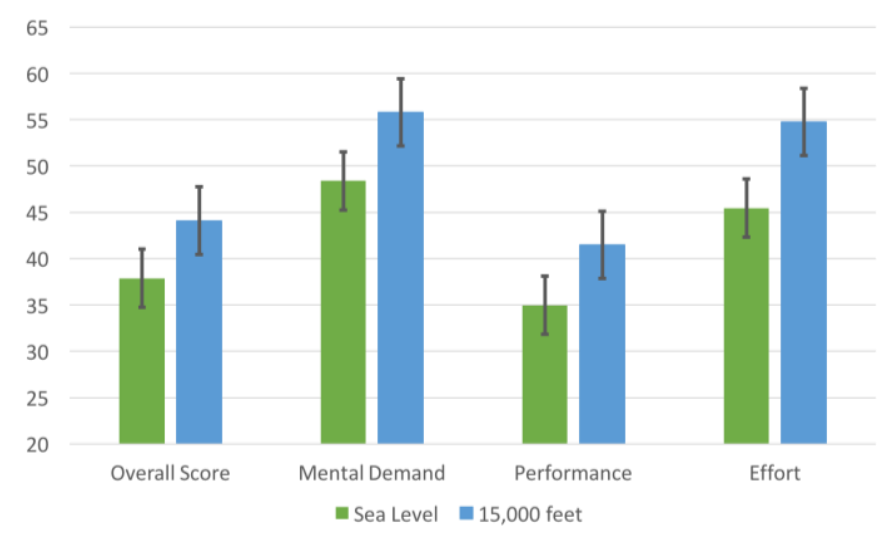

Figure 4. Self-reported workload NASA-TLX scores.

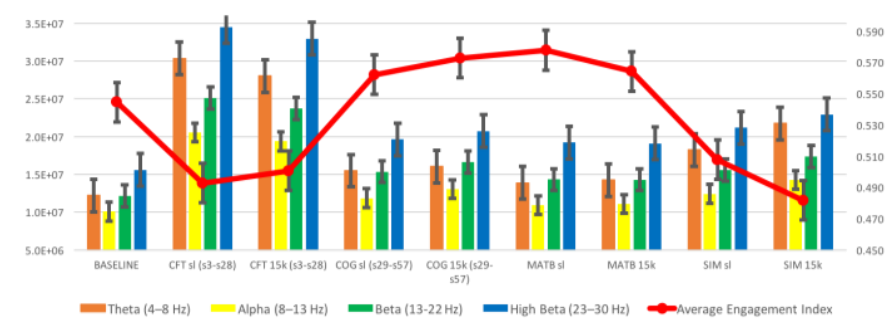

Figure 5. Total averaged EEG band powers and EI.

\section{DISCUSSION}

Results of this study indicated the effect of 15,000 feet simulated altitude did not induce performance decrement in the computer-based tasks (CFT, COG, MATB) nor the FLT task. These non-significant performance scores on the various tasks assessing cognitive capacity (CFT/COG), skill, rule, and knowledge-based task performance (MATB and FLT) indicates 10 minutes of exposure to 15,000 feet normobaric simulated altitude is ineffective at inducing mild cognitive impairment. However, the subjective report demonstrated an increased effort to maintain equivalent performance across a wide array of simple and complex tasks. The results indicate the need for further testing of mild normobaric hypoxia to establish this method as a safe and controlled induction of cognitive impairment in future studies at NASA LaRC or other facilities capable of supporting the use of hypoxia induction techniques. The results of further analyses will be presented in the final conference presentation.

These data suggest that the use of mild hypoxia as a method to induce workload effects was successful but the induced cognitive impairment during simulated flight and other tasks was insufficient for robust human-autonomous systems integration testing. Further analyses will be performed to assess current dataset for statistically significant differences between Sea Level and 15,000 feet conditions. Future investigations could include additional hypoxia inductions at higher simulated altitudes to induce cognitive impairment, increased workload, and task performance decrements.

Another important consideration is that of the psychophysical behaviors the test batteries and tasks included in the present study assessed. The tasks included in the present study relied on psychomotor speed and response time to indicate performance detriments. Neuropsychologial tests and tasks which are more demanding may have been more sensitive to the altitude manipulation. Such assessments should be considered in future studies involving mild normobaric hypoxia exposure to ensure diagnostically sensitive measures are employed.

Consideration for individual differences with respect to sensitivity to hypoxia should also be incorporated into future studies employing hypoxia exposure. Selecting for a subset of individuals who are susceptible to hypoxia could afford researchers the possibility of more effective impairment and means of efficiently testing autonomous systems designed to mitigate human error due to HSAs.

This study represents on-going work at NASA LaRC intending to add to the current knowledge of physical, cognitive, flight performance and pilot workload assessment to improve automation support and increase aviation safety. The developed testing method proved to be a valid and robust experimental method, but stronger hypoxia induction is necessary and is being pursued. These works are being developed to employ psychophysiologically-based inputs to automation to increase aviation safety, and specifically, for the development of increasingly autonomous systems.

\section{ACKNOWLEDGEMENTS}

The authors would like to acknowledge the following individuals and organizations for their support in completing the study described in the current paper: Lt. Col. Thomas Massa and LAFB Physiology Unit Staff, Lt. Col. Christopher Borchardt, Dr. Leroy Gross and the NASA LaRC Health Clinic Staff, NASA LaRC Institutional Review Board, NASA LaRC Safety Office, and Mary Carolyn Last.

\section{REFERENCES}

DeHart, R. L., \& Davis, J. R. (2002). Fundamentals of Aerospace Medicine: Translating Research into Clinical Applications (3rd ed.). Philadelphia: Lippincott Williams and Wilkins.

Federation Aviation Administration Advisory Circular AC 61-107B. Retrieved from Internet:

https://www.faa.gov/regulations_policies/advisory_circulars/index.cfm/g o/document.information/documentID/1020859

Gradwell, D. P. (2006). Hypoxia and hyperventilation. In D. J. Rainford \& D. P. Gradwell (Eds.), Ernsting's Aviation Medicine, (4th ed.), 51-54. Boca Raton, FL: CRC Press, Taylor \& Francis Group. 
Harding, R. M. \& Mills, F. J. (1983). Problems of altitude I: Hypoxia and hyperventilation. British Medical Journal, 286, 1408-1410.

Hart, S. G., \& Staveland, L. E. (1988). Development of NASA-TLX (Task Load Index): Results of empirical and theoretical research. In P. A. Hancock, \& N. Meshkati, Human mental workload (pp. 139-183). Oxford, England: North-Holland.

Hewett, K. J., Curry, I. P., Rath, E., \& Collins, S. M. (2009). Subtle cognitive effects of mild hypoxia. Fort Rucker, AL: U.S. Army Aeromedical Research Laboratory. USAARL Technical Report No. 2009-17.

Kay, G. (1995). CogScreen Aeromedical Edition Professional Manual. Washington DC: CogScreen LLC.

Nesthus, T. E., Rush, L. L. \& Wreggit, S. S. (1997). Effects of mild hypoxia on pilot performance at general aviation altitudes. U.S. Department of Transportation, Federal Aviation Administration Report DOT/FAA/AM97/9.

Nesthus, T. E., Rush, L. L. \& Wreggit, S. S. (1997). Effects of mild hypoxia on pilot performance at general aviation altitudes. U.S. Department of Transportation, Federal Aviation Administration Report DOT/FAA/AM$97 / 9$

Parasuraman, R. J., Molloy, R., \& Singh, I. L. (1993). Performance consequences of automation-induced "complacency.". International Journal of Aviation Psychology, 3(1), 1-23.

Pickard, J. S. (2002). The atmosphere and respiration. In: R. L. DeHart \& J. L. Davis (Eds.), Fundamentals of Aerospace Medicine (3rd ed.), 19-38. Philadelphia: Lippincott, Williams \& Wilkins.

Pope, A. T., \& Bogart, E. H. (1992). Identification of Hazardous Awareness States in Monitoring Environments. SAE Transactions: Journal of Aerospace, 101, 449-457.

Pope, A. T., Bogart, E. H., \& Bartolome, D. S. (1995). Biocybernetic System Validates Index of Operator Engagement in Automated Task. Biological Psychology, 40, 187-195.

Rasmussen, J. (1982). Human errors: A taxonomy for describing human malfunctions in industrial installations. Journal of Occupational Accidents, 4, 311-333.

Rice, G. M., Moore, J. L., Jernigan, C., Moore, J. L., Clemons, E., Rife, C., \& Kay, G. G. (2005). Cognitive performance at simulated altitudes of 10,000, 12,000, and 15,000 feet utilizing the Cogscreen-Hypoxia Edition (Cogscreen-HE) [Abstract]. Aviation, Space, and Environmental Medicine, 76(3), 231-232.

Scerbo, M. W. (1996). Theoretical perspectives on adaptive automation. In R. J. Parasuraman \& M. Mouloua (Eds.), Automation and human performance: Theory and applications (pp. 37-64). Mahwah, NJ: Erlbaum Associates Inc.

Self, D. A., Mandella, J. G. \& Burian, D. (2013). Physiological determinants of human acute hypoxia tolerance. Department of Transportation, Federal Aviation Administration. Technical Report DOT/FAA/AM$13 / 22$.

Stephens, C. L., Scerbo, M. W., \& Pope, A. T. (2012). Adaptive Automation for Mitigation of Hazardous States of Awareness. In G. Matthews, C. Neubauer, P. A. Desmond, \& P. A. Hancock (Eds.), The Handbook of Operator Fatigue. Farnham, Surrey, UK: Ashgate.

Swain, C. A., Kourtidou, C. \& Wilson, G. F. (1999). The effects of hypobaric hypoxia on psychophysiological measures of cognitive functioning and performance. Air Force Research Lab Technical Report, AFRL-HE-WPTR-1999-0152, pp. 1-45.

Temme, L. A., Still, D. L. \& Acromite, M. T. (2010). Hypoxia and flight simulator performance of military instructor pilots in a flight simulator. Aviation, Space, and Environmental Medicine, 81, 654-659.

United States Air Force Aerospace Physiology. (2008). Aerospace Physiology Training Chapter 6. Reference AFI 11-403.

Weaver, J., Bradley, K., Burke, K., Helmick, J., Greenwood-Ericksen, A., \& Hancock, P. (2003). An experimental investigation of skill, rule, and knowlege-based performance under noise conditions. Paper presented at the Human Factors and Ergonomics Society 47th Annual Meeting, Santa Monica, CA.

Westermann, R. A. (2004). Hypoxia familiarisation training by the reduced oxygen breathing method. Aviation Medicine, 5, 11-15.

Wickens, C. D., Hooey, B. L., Gore, B. F., Sebok, A., \& Koenicke, C. S. (2009). Identifying Black Swans in NextGen: Predicting Human Performance in Off-Nominal Conditions. Human Factors: The Journal of the Human Factors and Ergonomics Society, 51(5), 638-651.
Wolf, M. (2014). Physiological consequences of rapid or prolonged aircraft decompression: Evaluation using a human respiratory model. Aviation, Space and Environmental Medicine, 85, 466-472.

Woodrow, A. D. \& Webb, J. T. (2011). Chapter 3, Atmospheric Environmental Effects. In: Handbook of Aerospace Physiology. Air Force Research Lab Report AFRL-SA-WP-SR-2011-0003, p 3.1-3.43.

Yang J.H., Kennedy Q, Sullivan J, Fricker RD Jr. (2013) Pilot performance: assessing how scan patterns \& navigational assessments vary by flight expertise. Aviation, Space, and Environmental Medicine. 84(2):116-124. 